$$
N=a^{2}-b^{2}
$$

where

$$
a=\left(2^{3} \cdot 5 \cdot 3^{2} \cdot 53^{2}\right) x+11150802925 .
$$

This representation of $a$ can be deduced from theory presented by Kraitchik [1], combined with the fact that both -1 and 5 are quadratic residues of $N$, as established by suitable representations of $N$ by quadratic forms.

Corresponding to $x=102908, a^{2}-N$ is the square of $b=114674787084$. Hence, $N$ is the difference of the squares of $a=115215488845$ and of the preceding value of $b$. Thus

$$
N=540701761 \cdot 229890275929 .
$$

The primality of each of these factors was determined in a similar manner. The factorization of $2^{159}-1$ is, therefore, now complete.

19 Snape Street

Kingsford, New South Wales

1. M. Kraitchik, Théorie des Nombres, Gauthier-Villars et Cie, Paris, 1922, p. 146.

\title{
Two Formulas Relating to Elliptic Integrals of the Third Kind
}

\section{By J. Boersma}

Using Legendre's notation, the normal elliptic integral of the third kind is defined by the equation

$$
\Pi\left(\phi, \alpha^{2}, k\right)=\int_{0}^{\phi} \frac{d \theta}{\left(1-\alpha^{2} \sin ^{2} \theta\right) \sqrt{1-k^{2} \sin ^{2} \theta}} .
$$

For $k^{2}<1$, the following expansion holds uniformly over the closed interval $0 \leqq \theta \leqq \frac{\pi}{2}$ :

$$
\frac{1}{\sqrt{1-k^{2} \sin ^{2} \theta}}=\sum_{m=0}^{\infty}\left(\begin{array}{c}
-\frac{1}{2} \\
m
\end{array}\right)(-1)^{m} k^{2 m} \sin ^{2 m} \theta
$$

where $\left(\begin{array}{c}-\frac{1}{2} \\ m\end{array}\right)=\frac{\left(-\frac{1}{2}\right)\left(-\frac{3}{2}\right) \cdots\left(-\frac{1}{2}-m+1\right)}{m !}$ for $m>0$, and $\left(\begin{array}{c}-\frac{1}{2} \\ 0\end{array}\right)=1$.

The factor $\frac{1}{1-\alpha^{2} \sin ^{2} \theta}$ in the integrand is bounded for $-\infty<\alpha^{2}<\frac{1}{\sin ^{2} \phi}$ and $0 \leqq \theta \leqq \phi$; consequently, the expanded integrand may be integrated term by term. Such integration yields the series

$$
\prod\left(\phi, \alpha^{2}, k\right)=\sum_{m=0}^{\infty} b_{m} k^{2 m}
$$

Received May 16, 1960. 
where

$$
b_{m}=\left(\begin{array}{c}
-\frac{1}{2} \\
m
\end{array}\right)(-1)^{m} \int_{0}^{\phi} \frac{\sin ^{2 m} \theta}{1-\alpha^{2} \sin ^{2} \theta} d \theta, m>0
$$

and

$$
\begin{aligned}
b_{0}=\int_{0}^{\phi} \frac{d \theta}{1-\alpha^{2} \sin ^{2} \theta} & =\frac{1}{\sqrt{1-\alpha^{2}}} \tan ^{-1}\left[\sqrt{1-\alpha^{2}} \tan \phi\right], \text { for }-\infty<\alpha^{2}<1, \\
& =\tan \phi, \text { for } \alpha^{2}=1 \\
& =\frac{1}{\sqrt{\alpha^{2}-1}} \tanh ^{-1}\left[\sqrt{\alpha^{2}-1} \tan \phi\right], \text { for } 1<\alpha^{2}<\frac{1}{\sin ^{2} \phi} .
\end{aligned}
$$

In general, the coefficients $b_{m}$ satisfy the recurrence relation

$$
2(m+1) \alpha^{2} b_{m+1}=(-1)^{m+1}(2 m+1)\left(\begin{array}{c}
-\frac{1}{2} \\
m
\end{array}\right) t_{2 m}(\phi)+(2 m+1) b_{m}
$$

where $t_{2 m}(\phi)=\int_{0}^{\phi} \sin ^{2 m} \theta d \theta$.

Byrd and Friedman [1] give [formula (902.00)] the recurrence relation

$$
t_{2 m}(\phi)=\frac{2 m-1}{2 m} t_{2 m-2}(\phi)-\frac{1}{2 m} \sin ^{2 m-1} \phi \cos \phi
$$

and explicit expressions for $t_{0}(\phi), t_{2}(\phi)$, and $t_{4}(\phi)$. Corresponding to these we find

$$
\begin{aligned}
& b_{1}=\frac{b_{0}-\phi}{2 \alpha^{2}} \\
& b_{2}=\frac{1}{16 \alpha^{4}}\left[3 \alpha^{2} \sin \phi \cos \phi+6 b_{0}-3\left(2+\alpha^{2}\right) \phi\right] \\
& b_{3}=\frac{5}{128 \alpha^{6}}\left[2 \alpha^{4} \sin ^{3} \phi \cos \phi+\alpha^{2}\left(3 \alpha^{2}+4\right) \sin \phi \cos \phi+8 b_{0}-\left(8+4 \alpha^{2}+3 \alpha^{4}\right) \phi\right] .
\end{aligned}
$$

When $\phi=\frac{\pi}{2},-\infty<\alpha^{2}<1$, and $k^{2}<1$, we deduce the following expansion of the complete elliptic integral of the third kind:

$$
\Pi\left(\alpha^{2}, k\right) \equiv \Pi\left(\frac{\pi}{2}, \alpha^{2}, k\right)=\sum_{m=0}^{\infty} c_{m} k^{2 m},
$$

where

$$
\begin{aligned}
& c_{0}=\frac{\pi}{2 \sqrt{1-\alpha^{2}}} \\
& c_{1}=\frac{\pi}{4 \alpha^{2}}\left[\frac{1}{\sqrt{1-\alpha^{2}}}-1\right] \\
& c_{2}=\frac{3 \pi}{32 \alpha^{4}}\left[\frac{2}{\sqrt{1-\alpha^{2}}}-2-\alpha^{2}\right]
\end{aligned}
$$




$$
c_{3}=\frac{5 \pi}{256 \alpha^{6}}\left[-4 \alpha^{2}-3 \alpha^{4}-8+\frac{8}{\sqrt{1-\alpha^{2}}}\right]
$$

and, in general, the coefficients satisfy the recurrence formula

$$
2(m+1) \alpha^{2} c_{m+1}=-\left(m+\frac{1}{2}\right) \pi\left(\begin{array}{c}
-\frac{1}{2} \\
m
\end{array}\right)^{2}+(2 m+1) c_{m},
$$

which follows from the recurrence formula for $b_{m}$ when use is made of the definite integral

$$
\begin{aligned}
t_{2 m}\left(\frac{\pi}{2}\right)=\int_{0}^{\pi / 2} \sin ^{2 m} \theta d \theta & =\frac{1}{2} \cdot \frac{\Gamma\left(m+\frac{1}{2}\right) \Gamma\left(\frac{1}{2}\right)}{m !} \\
& =\frac{\pi}{2}(-1)^{m}\left(\begin{array}{c}
-\frac{1}{2} \\
m
\end{array}\right) .
\end{aligned}
$$

The expansions obtained above for $\Pi\left(\phi, \alpha^{2}, k\right)$ and $\Pi\left(\alpha^{2}, k\right)$ constitute extensions and simplifications of formulas (906.01) and (906.00), respectively, in the book already cited, by Byrd and Friedman. Furthermore, the coefficient of $\alpha^{2}$ has been corrected here in the expression for $c_{3}$ appearing in (906.00).

Mathematical Institute

University of Groningen

The Netherlands

1. Paul F. Brrd \& Morris D. Frieddan, Handbook of Elliptic Integrals for Engineers and Physicists, Springer-Verlag, Berlin, 1954. 